\title{
Chaperone-Like Effect of Polyzwitterions on the Interaction of C1q with IgG
}

\author{
Ivo Ivanov ${ }^{\mathrm{a}}$, Ivanka Tsacheva ${ }^{\mathrm{b}, *}$, Vishnia Stoyanova $^{\mathrm{b}}$, Miroslav Nikolov $^{\mathrm{b}}$, \\ Magdalena I. Tchorbadjieva ${ }^{\mathrm{b}}$, Svetla Petrova ${ }^{\mathrm{b}}$, Latchezar Christov ${ }^{\mathrm{a}}$, \\ Ventsislava Georgieva ${ }^{\mathrm{a}}$, and George Georgiev ${ }^{\mathrm{a}}$ \\ a Sofia University, Faculty of Chemistry, 1 James Bouchier Aven., 1164 Sofia, Bulgaria \\ b Sofia University, Faculty of Biology, Department of Biochemistry, 8 Dragan Tsankov St., \\ 1164 Sofia Bulgaria. Fax +359-2-865-6641. E-mail: itsacheva@biofac.sofia-uni.bg \\ * Author for correspondence and reprint requests \\ Z. Naturforsch. 64c, 149-154 (2009), received August 18/September 12, 2008
}

The amphiphilic polyzwitterion (PZ) poly(ethylene oxide-b- $N, N$-dimethyl(methacryloyloxyethyl)ammonium propanesulfonate), zwitterionic surfactant (ZS) n-dodecyl$N, N$-dimethyl-3-ammonium-1-propanesulfonate, and zwitterionic monomer (ZM) $N, N$ dimethyl(methacryloyloxyethyl)ammonium propanesulfonate were analyzed for their suggested chaperone-like effect on the interaction of $\mathrm{C} 1 \mathrm{q}$ and $\mathrm{IgG}$. Our results proved that the $\mathrm{PZ}$ retarded the $\mathrm{C1q}$ interaction with $\mathrm{IgG}$, demonstrating a specific protein-folding helper effect. The ZS enhanced this interaction, when the ZS concentration was lower than the critical micelle concentration $(C M C)$, and retarded it, when the ZS concentration was above the $C M C$. The ZM, with no self-assembling ability, did not influence this interaction. These results support the hypothesis of a hydrophobic interaction between Pts and hydrophobic domains of partly denatured protein molecules. The amphiphilic self-assemblies, formed by polyzwitterionic macromolecules or zwitterionic surfactants, have the ability to transform the hydrophobic domains of the protein molecules into hydrophilic ones, covering them with their hydrophilic parts.

Key words: C1q Complement Component, Immunoglobulin G, Polyzwitterion, Zwitterionic Surfactant

\section{Introduction}

The complement system is the major effector of the innate humoral immunity. The activation of complement is known to be triggered by three different pathways and results in killing of pathogens either by opsonization or by direct lysis. $\mathrm{C} 1 \mathrm{q}$, the recognition molecule of the classical complement pathway, binds to the Fc fragment of immunoglobulins ( $\mathrm{IgG}$ and $\mathrm{IgM}$ ) in immune complexes, thus activating the complement system (Kishore and Reid, 2000). The C1q recognition and binding of $\mathrm{IgG}$ have been a focus of research for a long time. The IgG-binding motif of $\mathrm{C} 1 \mathrm{q}$ was demonstrated to have a charged nature. Two basic residues within IgG are necessary for C1q binding (Burton et al., 1980; Duncan and Winter, 1988), and the binding efficiency is dependent on the ionic strength $(I)$, thus implying an electrostatic interaction between $\mathrm{C} 1 \mathrm{q}$ and IgG (Marques et al., 1993; Kaul and Loos, 1997; Nauta et al., 2003; Kojouharova et al., 2004). Alternatively, there is evidence for hydrophobic motifs involved in the interaction of $\mathrm{C} 1 \mathrm{q}$ with IgG and other ligands (Malmsten et al.,1996). To clear this inconsistency we studied the interaction of $\mathrm{C} 1 \mathrm{q}$ with $\mathrm{IgG}$ in the presence of the amphiphilic polyzwitterion (PZ) poly(ethylene oxide-b$N, N$-dimethyl(methacryloyloxyethyl)ammonium propanesulfonate), zwitterionic surfactant (ZS) $n$-dodecyl- $N, N$-dimethyl-3-ammonium-1-propanesulfonate, and zwitterionic monomer (ZM) $N, N$-dimethyl(methacryloyloxyethyl)ammonium propanesulfonate (Fig. 1).

Previously we proved that in $\mathrm{PZ}$ aqueous solution with high $I$ values, $\mathrm{PZ}$ macromolecules transform into PZ micelles (as a result of selfassembling), whose core is composed of a macromolecular backbone and segments between the zwitterionic charges in all of the side chains, while their shell consists of both charges together with their counterions (Georgiev et al., 2005, 2006; Atanasov et al., 2006). These PZ self-assemblies are of great importance for in vitro preservation of the biopolymer macromolecule native states 
PZ

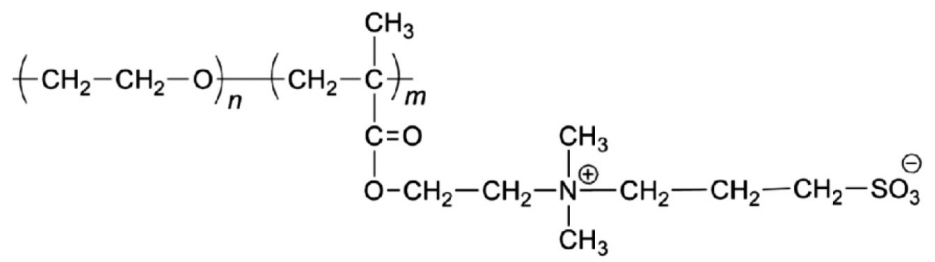

Poly(ethylene oxide-b- $N, N$-dimethyl(methacryloyloxyethyl)ammonium propanesulfonate)

ZM

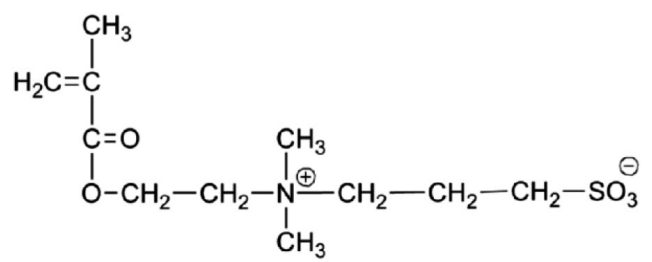

$N, N$-Dimethyl(methacryloyloxyethyl)ammonium propanesulfonate

$$
\begin{aligned}
& \text { ZS }
\end{aligned}
$$

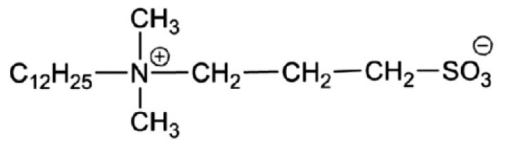

$$
\begin{aligned}
& n \text {-Dodecyl- } N, N \text {-dimethyl-3-ammonium-1-propanesulfonate }
\end{aligned}
$$

Fig. 1. Chemical structures of the PZ, ZM and ZS.

and cell membranes. This effect is a result of the interaction between the PZ micelle cores with the hydrophobic domains, exposed after perturbation of the native state of protein globules or cell membranes. When the hydrophobic cores interact with hydrophobic domains of the protein globule or cell membrane, the hydrophilic shell of the PZ micelle covers the hydrophobic "pocket" and the protein globule or membrane surfaces become hydrophilic again. This is just the transformation that hampers or prevents further undesired conformational transitions in the protein macromolecules, responsible for protein aggregation, as well as hydrophobic interactions, responsible for cell adhesion. Thus, due to this effect of PZ selfassemblies the PZs are considered to act as protein-folding helpers or chaperone-like macromolecules (Goldberg et al., 1996; Vuillard et al., 1998; Bezancon et al., 2003; Georgiev et al., 2006).

The binding of both hydrophilic and hydrophobic parts of the PZ macromolecules is of great importance for the $\mathrm{PZ}$ unique chaperone-like effect.
Free ZM molecules do not form such amphiphilic self-assemblies and therefore cannot act as chaperones. However, it is possible that a ZS could acquire such ability after micellization, when its concentration exceeds the critical micelle concentration $(C M C)$. The verification of these $\mathrm{PZ}, \mathrm{ZM}$ and $\mathrm{ZS}$ abilities to control the hydrophobic interaction between $\mathrm{C} 1 \mathrm{q}$ and $\mathrm{IgG}$ is the main objective of this work.

\section{Experimental}

\section{Materials}

$N, N$-Dimethyl(methacryloyloxyethyl)ammonium propanesulfonate (DMAPS) and monomethoxy ethyleneglycol with the molecular weight 2000 Da (Merck, Germany) were used without previous purification. All other reagents $(\mathrm{CuBr}$, $\mathrm{Na}_{2} \mathrm{HPO}_{4}, \mathrm{NaH}_{2} \mathrm{PO}_{4}, \mathrm{NaCl}$, Tween 20) of analytical grade were purchased from Merck (Germany).

The following buffers were used: PBS, phosphate-buffered saline $\left(0.01 \mathrm{M} \mathrm{Na}_{2} \mathrm{HPO}_{4}, 0.01 \mathrm{M}\right.$ 

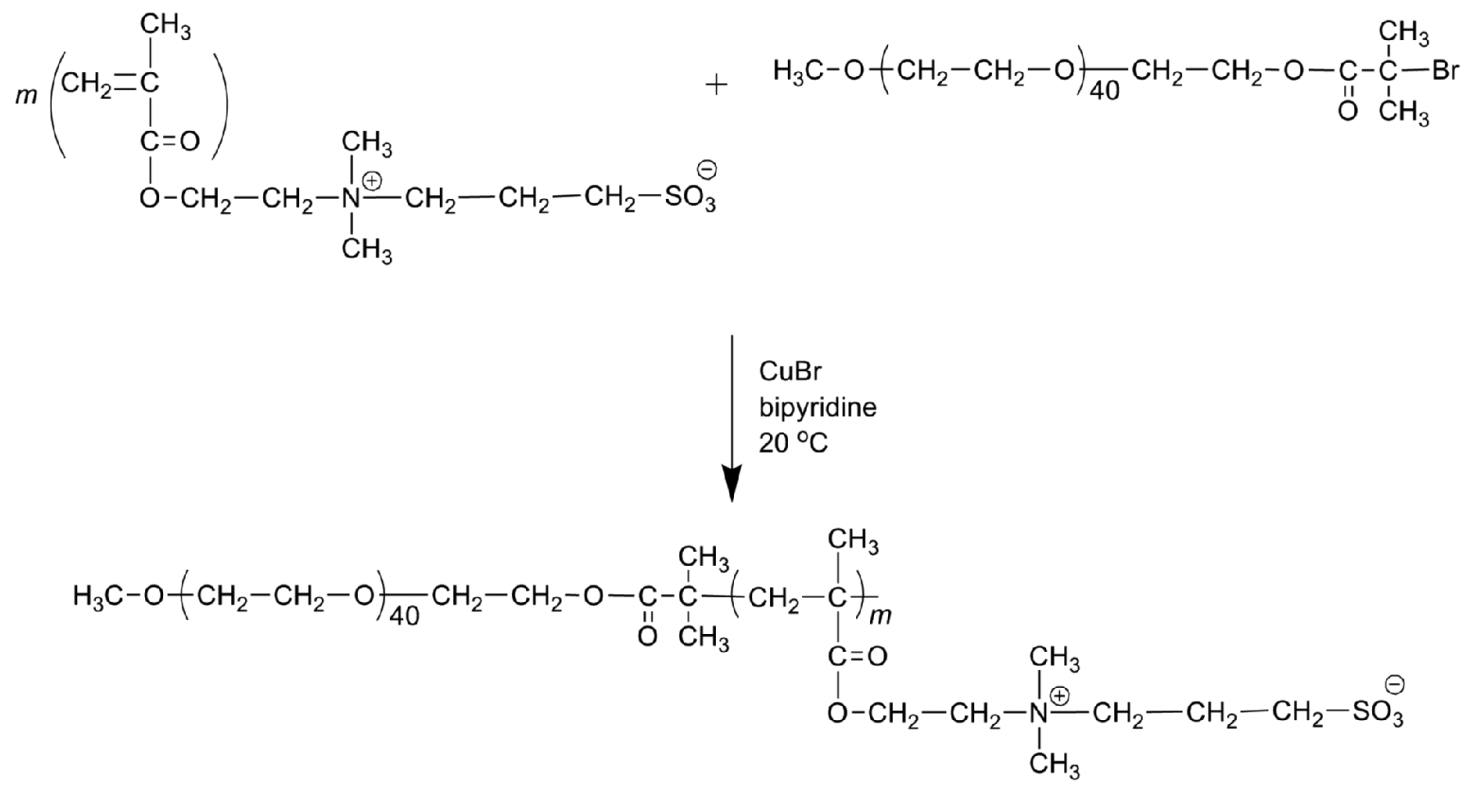

Fig. 2. Schematic presentation of the synthesis of the polyzwitterionic block-copolymer.

$\mathrm{NaH}_{2} \mathrm{PO}_{4}, 0.145 \mathrm{M} \mathrm{NaCl}, \mathrm{pH}$ 7.4); TPBS, PBS containing $0.05 \%$ Tween 20; AP, alkaline phosphatase buffer (100 mM Tris(hydroxymethyl)aminomethane, $150 \mathrm{~mm} \mathrm{NaCl}, 5 \mathrm{~mm} \mathrm{MgCl}_{2}$, $\mathrm{pH}$ 9.6).

C1q was isolated from human plasma by affinity chromatography on $\mathrm{IgG}-$ Sepharose, followed by affinity Protein G chromatography (Reid and Porter, 1976). The protein concentration was estimated by measuring the absorption at $280 \mathrm{~nm}$ and using the $A_{280}(1 \mathrm{t} \%, 1 \mathrm{~cm})$ coefficient of 6.82 . The purity of $\mathrm{C} 1 \mathrm{q}$ was assessed by SDS-PAGE (15 $1 \%$ ) under reducing conditions where it appeared as three bands, corresponding to the A, B, and $\mathrm{C}$ chains of 34,32 , and $27 \mathrm{kDa}$, respectively.

Heat-aggregated IgG (HAIgG) was used as a model for immune complexes.

\section{Synthesis of double hydrophilic copolymer}

First the oligoethylene oxide macroinitiator was synthesized as already described (Matyjaszewski and Xia, 2001). The macroinitiator was used for radical polymerization with atom transfer to produce the second polyzwitterionic block of copolymer according to Ma et al. (2002) (Fig. 2). The synthesized block-copolymer was dialyzed against water for $42 \mathrm{~h}$ and lyophilized.

\section{ELISA assays}

The principal scheme for the ELISA assays was as follows: The microtitre plates were coated with IgG $(20 \mu \mathrm{g} /$ well $)$ in PBS and the remaining binding sites were blocked with $1 \mathrm{t} \%$ BSA. Then the plates were incubated with $\mathrm{C} 1 \mathrm{q}(8 \mu \mathrm{g} /$ well) in PBS containing different concentrations of the $\mathrm{PZ}$ or $\mathrm{ZM}$ or $\mathrm{ZS}$. The bound human $\mathrm{C} 1 \mathrm{q}$ was detected by subsequent incubations with rabbit polyclonal anti-C1q (DAKO) and anti-rabbit IgG-alkaline phosphatase conjugate (Sigma). After the incubations the enzyme reaction was carried out with $p$-nitrophenyl phosphate dissolved in AP. The absorbance was read at $405 \mathrm{~nm}$. All the incubations were carried out for $1 \mathrm{~h}$ at $37^{\circ} \mathrm{C}$. After each incubation the wells were washed three times with TPBS.

\section{Results and Discussion}

The effect of PZs, ZMs and ZSs on the interaction of $\mathrm{C} 1 \mathrm{q}$ with IgG was analyzed by ELISA. The results for the effect of PZs, assessed at different concentrations of zwitterionic monomer units $\left(C_{\mathrm{PZ}}, \mathrm{mu}=0.05,1.50,25.00\right.$ and $\left.50.00 \mathrm{~mm}\right)$ are shown in Fig. 3. The comparison between $A_{405 \mathrm{~nm}}$ as a measure of the $\mathrm{IgG}-\mathrm{C} 1 \mathrm{q}$ interaction in the presence of $\operatorname{IgG}\left(A_{\mathrm{S}}\right)$ and in the absence of $\operatorname{IgG}\left(A_{\mathrm{C}}\right)$ 


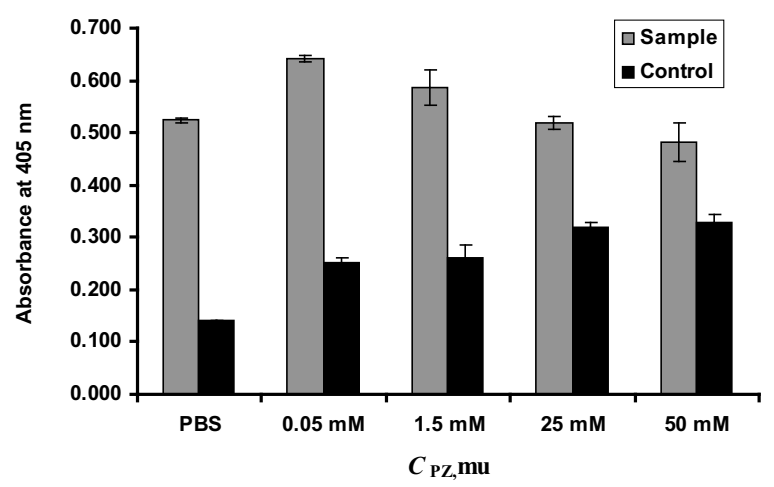

Fig. 3. ELISA assay for IgG binding of human $\mathrm{C} 1 \mathrm{q}$ in the presence of different PZ monomer unit concentrations $\left(C_{\mathrm{PZ}}, \mathrm{mu}\right)$. C1q $(8 \mu \mathrm{g} /$ well $)$ in PBS containing different concentrations of the PZ was added to IgG-coated plates $(20 \mu \mathrm{g} / \mathrm{well})$. The plates were incubated for $1 \mathrm{~h}$ at $37^{\circ} \mathrm{C}$ and rabbit anti-human $\mathrm{C} 1 \mathrm{q}$ antibody $(1 \mu \mathrm{g} / \mathrm{well})$ was added and incubated again for $1 \mathrm{~h}$. The amount of bound $\mathrm{C} 1 \mathrm{q}$ was visualized by alkaline phosphatase conjugated to goat anti-rabbit IgG antibodies.

is presented as the ratio between $A_{\mathrm{S}} / A_{\mathrm{C}}$ at a given concentration of $\mathrm{PZ}$ monomer units $\left(C_{\mathrm{PZ}}, \mathrm{mu}\right)$ and $A_{\mathrm{S}} / A_{\mathrm{C}}$ in the absence of them: $g_{\mathrm{N}}=\left[A_{\mathrm{S}} / A_{\mathrm{C}}\right]_{\mathrm{PZ}} /\left[A_{\mathrm{S}} /\right.$ $\left.A_{\mathrm{C}}\right]_{0}$ (Table I). The results show that $g_{\mathrm{N}}$ decreases monotonically as the $C_{\mathrm{PZ}}$, mu increases, which is in full agreement with our expectation for a chaperone-like effect due to the PZ self-assemblies in the buffer aqueous solutions.

The results for the interaction of $\mathrm{C} 1 \mathrm{q}$ with $\mathrm{IgG}$ in the presence of $\mathrm{ZMs}$ indicate that the $\mathrm{ZM}$ concentration did not influence the $g_{\mathrm{N}}$ ratio, confirming that free $\mathrm{ZM}$ molecules do not have the ability to form amphiphilic self-assemblies and to take part in the retardation of the hydrophobic C1q interaction with IgG (Table I).

Notably representative are the results for the hydrophobic interaction between $\mathrm{Clq}$ and $\mathrm{IgG}$ in the presence of ZSs (Table I). There are two essential characteristic features of this " $g_{\mathrm{N}}-C_{\mathrm{ZS}}$ "

Table I. Comparison between the effects of the PZ, ZM and $\mathrm{ZS}$ on the interaction of $\mathrm{Clq}$ with $\mathrm{IgG}$ in terms of $g_{\mathrm{N}}$ values.

\begin{tabular}{lccc}
\hline Concentration & $g_{\mathrm{N}, \mathrm{PZ}}$ & $g_{\mathrm{N}, \mathrm{ZM}}$ & $g_{\mathrm{N}, \mathrm{ZS}}$ \\
\hline PBS (control) & 1.000 & 1.000 & 1.000 \\
$0.05 \mathrm{mM}$ & 0.683 & 1.224 & 1.269 \\
$1.5 \mathrm{mM}$ & 0.602 & 0.933 & 1.716 \\
$25 \mathrm{mM}$ & 0.436 & 0.908 & 1.256 \\
$50 \mathrm{mM}$ & 0.393 & 1.026 & 0.758 \\
\hline
\end{tabular}
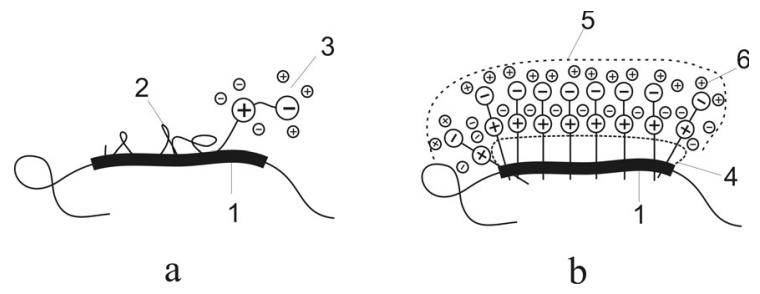

Fig. 4. Schematic presentation of the coating of the hydrophobic domain ( $\mathrm{C} 1 \mathrm{q}$ or $\mathrm{IgG})$ (a) with an isolated $\mathrm{ZS}$ molecule $\left(C_{\mathrm{ZS}}<C M C\right)$, when the hydrophobic tail effect dominates over the ZS molecule head, and (b) with the $\mathrm{ZS}$ micelle $\left(C_{\mathrm{Zs}}>C M C\right)$, when the hydrophilic micelle shell covers totally the hydrophobic domain. 1, Hydrophobic domain of the protein molecule; 2, ZS molecule hydrophobic tail; 3 , ZS molecule hydrophilic head; 4, hydrophobic ZS micelle core; 5, hydrophobic ZS micelle shell; 6 , counterion atmosphere.

relationship: (i) The considerable augmentation of the C1q-IgG interaction at low ZS concentration and its retardation at high ones; (ii) its positive maximum. This more complex dependence could be attributed to the influence of the individual ZS micelles at low and high ZS concentrations, when $C_{\mathrm{Zs}}>C M C_{\mathrm{zs}}$. In fact, if $C_{\mathrm{Zs}}<$ $C M C_{\mathrm{ZS}}$, the hydrophobic domains of the free $\mathrm{ZS}$ molecules absorbed on the $\mathrm{C} 1 \mathrm{q}$ or $\mathrm{IgG}$ enhance the hydrophobic interaction due to the dominance of the ZS hydrophobic tail effect over the ZS head (Fig. 4a). The increase of the hydrophobic surface due to the long hydrophobic tail of the ZS molecule is far greater than the decrease of this surface due to the hydrophilic molecule head. The higher ZS concentration should induce a stronger hydrophobic interactions between $\mathrm{C} 1 \mathrm{q}$ and IgG. However, the situation changes drastically after reaching $C M C_{\mathrm{ZS}}$. The $\mathrm{ZS}$ micelle formation covers the protein hydrophobic domain, totally transforming its hydrophobic surface into a hydrophilic one due to the micelle hydrophilic shell (Fig. 4b). As a result of this transition, the hydrophobic $\mathrm{C} 1 \mathrm{q}$ interaction with $\mathrm{IgG}$ decreases gradually, and at higher ZS concentrations (high ZS micelle concentration) the retardation of this interaction becomes similar to that in the presence of PZs in solution. The $g_{\mathrm{N}}$ value becomes negative again. This similarity is a result of the fact that in both cases large amphiphilic selfassemblies are produced, and their effect on the protein hydrophobic surface is identical: transformation of the hydrophobic surface into a hy- 
drophilic one. The very fine difference between both effects lies in the PZ self-assemblies formed at any PZ concentration (due to the binding of the zwitterionic monomer units), while the ZS micelle formation is a concentration-dependent self-assembling process and ZS micelles are only produced at ZS concentrations higher than $C M$ $C_{\mathrm{Zs}}$. This is the reason for the difference between the $g_{\mathrm{N}}$ vs. $C_{\mathrm{PZ}}$ and $g_{\mathrm{N}}$ vs. $C_{\mathrm{ZS}}$ values.

\section{Conclusion}

Our results proved that the $\mathrm{PZ}$ retarded the interaction of $\mathrm{C} 1 \mathrm{q}$ with $\mathrm{IgG}$, demonstrating a specific protein-folding helper effect. On the other hand, the $\mathrm{ZS}$ enhanced this interaction if the $\mathrm{ZS}$ concentration was lower than the $C M C$, and retarded it when the ZS concentration was higher than the $C M C$. The ZM, with no self-assembling ability, did not influence this interaction.

After the first confirmation of the PZ proteinfolding helper ability (Vuillard et al., 1998; Bezancon et al., 2003; Georgiev et al., 2006), the results presented above support the hypothesis of hydrophobic interaction between the PZ and hy-

Atanasov V., Vassileva E., Kamenova I., Ivanov I., and Georgiev G. (2006), Double hydrophilic block zwitterionic copolymer as an acid phosphatase folding helper. Ann. Univ. Sofia Chem. 98-99, 317-325.

Bezancon N., Rabilloud T., Vuillard L., and Goldberg M. (2003), Physical-chemical features of non-detergent sulfobetaines active as protein-folding helpers. Biophys. Chem. 100, 469-479.

Burton D. R., Boyd J., Brampton A. D., EasterbrookSmith S. B., Emanuel E. J., Novotny J., Redmatcher T. W., van Schravendijk M. R., and Dwek R. A. (1980), The Clq receptor site on immunoglobulin G. Nature 288, 338-344.

Duncan A. R. and Winter G. (1988), The binding site for C1q on IgG. Nature 332, 738-740.

Georgiev G., Kamenska E., Vassileva E., Kamenova I., Georgieva V., Iliev S., and Ivanov I. (2005), Properties and biomedical application of polyzwitterions. Ann. Univ. Sofia Chem. 98-99, 305-325.

Georgiev G., Kamenska E., Vassileva E., Kamenova I., Georgieva V., Iliev S., and Ivanov I. (2006), Selfassembly, antipolyelectrolyte effect, nonbiofouling properties of polyzwitterions. Biomacromolecules 7, 1329-1334.

Goldberg M., Bezancon N., Vuillard L., and Rabilloud T. (1996), Non-detergent sulfobetaines: A new class of molecules that facilitate in vitro protein renaturation. Folding Des. 1, 21-27. drophobic domains of partially denatured protein molecules (C1q, IgG, enzymes). The amphiphilic self-assemblies, formed by PZ macromolecules or the ZS, have the ability to transform the hydrophobic domains of the protein molecules into hydrophilic ones, covering them with their hydrophilic parts. The self-assembling of the PZ macromolecules is not a concentration-dependent process because of the binding of the zwitterionic monomer units within the polymer chain, while the ZS self-association is a concentration-dependent process and takes place only when the surfactant concentration is higher than the $C M C$. This different behaviour is the reason for the various effects of a PZ and ZS on the interaction of $\mathrm{C} 1 \mathrm{q}$ with $\mathrm{IgG}$, shown here for the first time. At the same time, our results confirm the contribution of hydrophobic motifs of $\mathrm{C} 1 \mathrm{q}$ involved in its interaction with $\mathrm{IgG}$.

\section{Acknowledgement}

The authors are grateful for financial support by EC, 6FP (Grant № NMP4-ct-2007-033168) and the Sofia University Science Fund (Grant № 61/2008).

Kaul M. and Loos M. (1997), Dissection of C1q capability of interaction with IgG: time-dependent formation of a tight and only partly reversible association. J. Biol. Chem. 272, 33234-33244.

Kishore U. and Reid K. B. M. (2000), C1q: structure, function and receptors. Immunopharmacology $\mathbf{4 2}$, $159-163$.

Kojouharova M. S., Gadjeva M. G., Tsacheva I. G., Zlatarova A. S., Roumenina L. T., Tchorbadjieva M. I., Atanasov B. P., Waters P., Urban B. C., Sim R. B., Reid K. B. M., and Kishore U. (2004), Mutational analyses of the recombinant globular regions of human $\mathrm{C} 1 \mathrm{q} \mathrm{A}, \mathrm{B}$, and $\mathrm{C}$ chains suggest an essential role for arginine and histidine residues in the $\mathrm{C} 1 \mathrm{q}-\mathrm{IgG}$ interaction. J. Immunol. 172, 4351-4358.

Ma I. Y., Lobb E. J., Billingham N. C., Armes S. P., Lewis A. L., Lloyd A. W., and Salvage J. (2002), Synthesis of biocompatible polymers. 1 . Homopolymerization of 2-methacryloyloxyethyl phosphorylcholine via ATRP in protic solvents: An optimization study. Macromolecules 35, 9306-9314.

Malmsten M., Lassen B., Van Alstine J. M., and Nilsson R. U. (1996), Adsorption of complement proteins C3 and C1q. J. Coll. Interf. Sci. 178, 123-134.

Marques G., Anton L. C., Barrio E., Sanchez A., Ruiz S., Gavilanes F., and Vivanco F. (1993), Arginine residues of the globular regions of human $\mathrm{C} 1 \mathrm{q}$ involved in the interaction with immunoglobulin G. J. Biol. Chem. 268, 10393-10402. 
Matyjaszewski K. and Xia J. H. (2001), Atom transfer radical polymerization. Chem. Rev. 101, 2921-2990.

Nauta A. J., Bottazzi B., Mantovani A., Salvatori G., Kishore U., Schwaeble W. J., Gingras A. R., Tzima S., Vivanco F., Egido J., Tijsma O., Hack E.C., Daha M.R., and Roos A. (2003), Biochemical and functional characterization of the interaction between pentraxin 3 and C1q. Eur. J. Immunol. 33, 465-473.
Reid K. B. M. and Porter R. R. (1976), Subunit composition and structure of subcomponent C1q of the first component of human complement. Biochem. J. $\mathbf{1 5 5}, 19-23$.

Vuillard L., Rabilloud T., and Goldberg M. (1998), Interactions of non-detergent sulfobetaines with early folding intermediates facilitate in vitro protein renaturation. Eur. J. Biochem. 256, 128-135. 